\title{
Computational Simulations; Abundant Optical Wave Solutions Atangana Conformable Fractional Nonlinear Schrödinger Equation
}

\author{
Mostafa M. A. Khater $\mathbb{D}^{1,},{ }^{1,2}$ Mustafa Inc $\mathbb{D}^{3},{ }^{3,4,5}$ Raghda A. M. Attia, ${ }^{6,7}$ and Dianchen Lu $\mathbb{I D}^{1}$ \\ ${ }^{1}$ Department of Mathematics, Faculty of Science, Jiangsu University, Zhenjiang 212013, China \\ ${ }^{2}$ Department of Basic Science, Obour High Institute for Engineering and Technology, Cairo 11828, Egypt \\ ${ }^{3}$ Department of Computer Engineering, Biruni University, Istanbul, Turkey \\ ${ }^{4}$ Science Faculty, Firat University, Elazig 23119, Turkey \\ ${ }^{5}$ Department of Medical Research, China Medical University Hospital, China Medical University, Taichung, Taiwan \\ ${ }^{6}$ Department of Basic Science, Higher Technological Institute 10th of Ramadan City, El Sharqia 44634, Egypt \\ ${ }^{7}$ School of Management \& Economics, Jiangsu University of Science and Technology, Zhenjiang 212003, China
}

Correspondence should be addressed to Mostafa M. A. Khater; mostafa.khater2024@yahoo.com and Mustafa Inc; minc@ firat.edu.tr

Received 28 May 2021; Accepted 6 January 2022; Published 10 February 2022

Academic Editor: Jorge E. Macias-Diaz

Copyright $(92022$ Mostafa M. A. Khater et al. This is an open access article distributed under the Creative Commons Attribution License, which permits unrestricted use, distribution, and reproduction in any medium, provided the original work is properly cited.

\begin{abstract}
This research paper explores the Atangana conformable nonlinear fractional Schrödinger equation's optical soliton wave solutions through three recently introduced computational schemes. The simplest expanded equation, the generalized Kudryashov method, and the sech-tanh expansion approaches are used for describing the structure of optical solitons by nonlinear optical fibers with the modern fractional operator. Several formulas such as hyperbolic, trigonometric, logical, dim, light, moon-bright hybrid, singular, combined singular, and regular wave solutions have been created. The employed methods are effective and worthy of being tested. The features of the Hamiltonian process were used to analyze the stability properties of the solutions obtained.
\end{abstract}

\section{Introduction}

The nonlinear partial differential equation is one of the most exciting science types, as it can describe several nonlinear phenomena as nonlinear partial distinctiveness equations (NLPD) or a form of NLPD [1-3], including diffusion, heat, electrostatics, fluid dynamics, electrodynamic, elasticity, and quantum mechanics. Some equations include the uncertain functions of multivariable and partial byproducts, including integral and partial derivatives $[4,5]$. Several investigators in various fields have been studying the fractional order in the NLPD equations [6]. This analysis is focused on the nonlocal properties that only occur in such a derivative, as they are not present in an integer derivative [7-9]. This property is generally used to describe a location, quantity, and nonlocal
Lagrangian behavior at a distance [10]. According to this property, various forms of concepts are employed to transform the NLPD equation into a standard differential equation with an integer order, including Riemann-Liouville, the two-scale fractal derivative, He fractional derivative, Caputo, and compliant fractional derivatives [11-13]. The compliant fractional derivative of Atangana is used here on the grounds of its supremacy over other concepts of fractional derivatives $[14,15]$. This superiority is apparent in its capacity to extend all organizational characteristics, such as a chain law, quotient law, semigroup property, and element rule, to the traditional first derivative $[16,17]$.

Many analytical and semianalytical schemes are derived for the same purpose, such as Lie group method, $\left(G^{\prime} / G\right)$-expansion method, tanh-expansion method, 
extended tanh-expansion method, improved Bernoulli subequation function, Riccati-Bernoulli sub-ODE method, sinh-cosh method, simplest equation method, generalized Kudryashov method, Adomian decomposition method, homotopy perturbation method, general homotopy analysis method, and so on [18-25].

The rest of the paper's sections are ordered as follows: Section 2 studies the performance of the extended simplest equation [26, 27], the generalized Kudryashov [28, 29], and the sech-tanh expansion methods $[30,31]$ on the perturbed time-fractional nonlinear Schrödinger equation. Moreover, we study the stability property of obtained analytical wave solutions. Section 3 represents some of the obtained solutions in three \& two-dimensional and contour plots. Section 4 gives the conclusion.

\section{Application}

In this section, we apply the Atangana conformable fractional operator to the perturbed time-fractional nonlinear Schrödinger equation that is given by [31-35].

$$
\begin{aligned}
& i \frac{\partial^{\vartheta} \mathscr{F}}{\partial t^{\vartheta}}+\mathscr{F}_{x x}+\gamma \mathscr{F}|\mathscr{F}|^{2}+i\left[\gamma_{1} \mathscr{F}_{x x x}+\gamma_{2}|\mathscr{F}|^{2} \mathscr{F}_{x}\right. \\
& \left.+\gamma_{3}\left(|\mathscr{F}|^{2}\right)_{x} \mathscr{F}\right]=0,
\end{aligned}
$$

where $t>0,0<\vartheta<1, \quad i=\sqrt{-1}$, while $\alpha, \gamma$ are the arbitrary constants. Also, $\gamma_{1}$ represents the dispersion term, $\gamma_{2}$ is the nonlinear dispersion, $\gamma_{3}$ is the nonlinear dispersion term and $\mathscr{F}=\mathscr{F}(x, t)$ describes the propagation of optical solitons in optical fibers that exhibits a Kerr law nonlinearity. During the evolution age of communications, many fundamentals' applications are related to equation (1) such as plasma physics and optical fiber communications. This fiber is used to transmit light between the two ends of the fiber and the widespread use in fiber-optical links. They provide a broader and higher range of communication (data) than electrical cables. Typically crafted from drawing glass (silica) or plastic of a much thicker diameter than a human scalp.

Handling equation (1) via the Atangana conformable fractional in the next wave transformation $\mathscr{F}(x, t)=e^{i(h x-\Omega t)} \mathscr{G}(\mathcal{Z}), \quad \boldsymbol{Z}=x-c / \vartheta(t+(1 / \Gamma(\vartheta)))^{\vartheta}$ where $c$ is an arbitrary constant, yields

$$
\begin{aligned}
& e^{i(h x-t \Omega)}\left(\mathscr{G}(\boldsymbol{Z})\left(-h^{2}+\Omega+h^{3} \gamma_{1}\right)+\mathscr{G}^{3}(\boldsymbol{3})\left(\gamma-h \gamma_{2}\right)\right. \\
& -i\left(c-2 h+3 h^{2} \gamma_{1}\right) \mathscr{G}^{\prime(3)}+i \mathscr{G}^{2}(\boldsymbol{Z})\left(\gamma_{2}+2 \gamma_{3}\right) \mathscr{G}^{\prime(3)} \\
& \left.+\left(1-3 h \gamma_{1}\right) \mathscr{G}^{\prime \prime}(3)+i \gamma_{1} \mathscr{G}^{\prime \prime \prime}(\boldsymbol{Z})\right)=0 .
\end{aligned}
$$

Separating the real and imaginary part of equation (2) and integrating the imaginary part once with zero constant of integration lead to

$$
\begin{gathered}
\left(-h^{2}+\Omega+h^{3} \gamma_{1}\right) \mathscr{G}+\left(\gamma-h \gamma_{2}\right) \mathscr{G}^{3}+\left(1-3 h \gamma_{1}\right) \mathscr{G}^{\prime \prime}=0 \\
-\left(c-2 h+3 h^{2} \gamma_{1}\right) \mathscr{G}+\frac{\left(\gamma_{2}+2 \gamma_{3}\right)}{3} \mathscr{G}^{3}+\gamma_{1} \mathscr{G}^{\prime \prime}=0 .
\end{gathered}
$$

Comparing the coefficients of $\mathscr{G}, \mathscr{G}^{\prime \prime}, \mathscr{G}^{3}$ in equations (3) and (4) explains the equivalence between both equations under the following constraint

$$
\frac{-h^{2}+\Omega+h^{3} \gamma_{1}}{-\left(c-2 h+3 h^{2} \gamma_{1}\right)}=\frac{3\left(\gamma-h \gamma_{2}\right)}{\gamma_{2}+2 \gamma_{3}}=\frac{1-3 h \gamma_{1}}{\gamma_{1}} \text {. }
$$

This relation leads to $\Omega=-c+2 h+3 c h \gamma_{1}-8 h^{2} \gamma_{1}+$ $8 h^{3} \gamma_{1}^{2} / \gamma_{1}, \gamma_{2}=3 \gamma \gamma_{1}-2 \gamma_{3}+6 h \gamma_{1} \gamma_{3}$. Applying the homogenous balance rule to equation (4) for determining the balance between the nonlinear and highest order derivative terms.

2.1. Extended Simplest Equation Method. Applying this scheme to equation (4) leads to the following general solution

$$
\mathscr{G}(\boldsymbol{Z})=\sum_{i=-n}^{n} a_{i} f(3)^{i}=\frac{a_{-1}}{f(3)}+a_{0}+f(3) a_{1},
$$

where $a_{i},(i=-1,0,1)$ are arbitrary constants. Also, $f(3)$ satisfies the next auxiliary equation $[26,27]$

$$
f^{\prime}(\boldsymbol{Z})=\alpha+\lambda f(3)+\mu f(3)^{2},
$$

where $\alpha, \lambda, \mu$ are arbitrary constants. Substituting equations (6) along (7) into equation (4) and collecting all terms with the same power of $f(3)^{j},(j=-3,-2, \ldots, 2,3)$ lead to a system of algebraic equations. Solving this system yields the following families.

\subsubsection{Family $I$}

$$
\begin{aligned}
a_{0} & =\frac{\sqrt{3 / 2} \lambda \sqrt{\gamma_{1}}}{\sqrt{-\gamma_{2}-2 \gamma_{3}}}, a_{-1}=0, a_{1}=\frac{\sqrt{6} \mu \sqrt{\gamma_{1}}}{\sqrt{-\gamma_{2}-2 \gamma_{3}}}, c \\
& =\frac{1}{2}\left(4 h-6 h^{2} \gamma_{1}-\lambda^{2} \gamma_{1}+4 \alpha \mu \gamma_{1}\right),
\end{aligned}
$$

where $\left(-\gamma_{2}-2 \gamma_{3}>0, \gamma_{1}>0\right)$.

\subsubsection{Family II}

$$
\begin{aligned}
a_{0} & =\frac{\sqrt{3 / 2} \lambda \sqrt{\gamma_{1}}}{\sqrt{-\gamma_{2}-2 \gamma_{3}}}, a_{-1}=\frac{\sqrt{6} \alpha \sqrt{\gamma_{1}}}{\sqrt{-\gamma_{2}-2 \gamma_{3}}}, a_{1} \\
& =0, c=\frac{1}{2}\left(4 h-6 h^{2} \gamma_{1}-\lambda^{2} \gamma_{1}+4 \alpha \mu \gamma_{1}\right),
\end{aligned}
$$

where $\left(-\gamma_{2}-2 \gamma_{3}>0, \gamma_{1}>0\right)$.

Thus, the optical solitary wave solutions of the Atangana conformable fractional nonlinear Schrödinger equation are given based on family I as follows:

When $\lambda=0$,

for $\alpha \mu>0$, 


$$
\begin{aligned}
& \mathscr{F}_{1}(x, t)=\frac{\sqrt{6} e^{i h x-i t \Omega} \sqrt{\alpha \mu} \sqrt{\gamma_{1}} \operatorname{Tan}\left[\sqrt{\alpha \mu}\left(x+\theta+(t+1 / \Gamma(\vartheta))^{9}\left(-4 h+\left(6 h^{2}-4 \alpha \mu\right) \gamma_{1}\right) / 2 \vartheta\right)\right]}{\sqrt{-\gamma_{2}-2 \gamma_{3}}}, \\
& \mathscr{F}_{2}(x, t)=\frac{\sqrt{6} e^{i h x-i t \Omega} \sqrt{\alpha \mu} \operatorname{Cot}\left[\sqrt{\alpha \mu}\left(x+\theta+(t+1 / \Gamma(\vartheta))^{9}\left(-4 h+\left(6 h^{2}-4 \alpha \mu\right) \gamma_{1}\right) / 2 \vartheta\right)\right] \sqrt{\gamma_{1}}}{\sqrt{-\gamma_{2}-2 \gamma_{3}}} .
\end{aligned}
$$

For $\alpha \mu<0$,

$$
\begin{gathered}
\mathscr{F}_{3}(x, t)=\frac{\sqrt{6} \mathrm{e}^{\mathrm{i} h x-\mathrm{i} t \Omega} \sqrt{-\alpha \mu} \sqrt{\gamma_{1}} \operatorname{Tanh}\left[\sqrt{-\alpha \mu}\left(x+(t+1 / \Gamma(\vartheta))^{\vartheta}\left(-4 h+\left(6 h^{2}-4 \alpha \mu\right) \gamma_{1}\right) / 2 \vartheta\right) \mp \log [\theta] / 2\right]}{\sqrt{-\gamma_{2}-2 \gamma_{3}}}, \\
\mathscr{F}_{4}(x, t)=\frac{\sqrt{6} \mathrm{e}^{\mathrm{i} h x-\mathrm{i} t \Omega} \sqrt{-\alpha \mu} \operatorname{Coth}\left[\sqrt{-\alpha \mu}\left(x+(t+1 / \Gamma(\vartheta))^{\vartheta}\left(-4 h+\left(6 h^{2}-4 \alpha \mu\right) \gamma_{1}\right) / 2 \vartheta\right) \mp \log [\theta] / 2\right] \sqrt{\gamma_{1}}}{\sqrt{-\gamma_{2}-2 \gamma_{3}}} .
\end{gathered}
$$

When $\alpha=0$;

For $\lambda>0$

$$
\mathscr{F}_{5}(x, t)=-\frac{\sqrt{3 / 2} \mathrm{e}^{\mathrm{i} h x-\mathrm{i} t \Omega} \lambda \sqrt{\gamma_{1}}}{\left(-1+\mathrm{e}^{\lambda\left(x+\theta+(t+1 / \Gamma(9))^{9}\left(-4 h+\left(6 h^{2}+\lambda^{2}\right) \gamma_{1}\right) / 29\right)} \mu\right) \sqrt{-\gamma_{2}-2 \gamma_{3}}}-\frac{\sqrt{3 / 2} \mathrm{e}^{\mathrm{i} h x-\mathrm{i} t \Omega+\lambda\left(x+\theta+(t+1 / \Gamma(9))^{9}\left(-4 h+\left(6 h^{2}+\lambda^{2}\right) \gamma_{1}\right) / 29\right)} \lambda \mu \sqrt{\gamma_{1}}}{\left(-1+\mathrm{e}^{\lambda\left(x+\theta+(t+1 / \Gamma(9))^{9}\left(-4 h+\left(6 h^{2}+\lambda^{2}\right) \gamma_{1}\right) / 29\right)} \mu\right) \sqrt{-\gamma_{2}-2 \gamma_{3}}} .
$$

For $\lambda<0$

$$
\mathscr{F}_{6}(x, t)=\frac{\sqrt{3 / 2} e^{i h x-i t \Omega} \lambda \sqrt{\gamma_{1}}}{\sqrt{-\gamma_{2}-2 \gamma_{3}}}-\frac{\sqrt{6} e^{i h x-i t \Omega} \mu \sqrt{\gamma_{1}}}{\sqrt{-\gamma_{2}-2 \gamma_{3}}}+\frac{\sqrt{6} e^{i h x-i t \Omega} \mu \sqrt{\gamma_{1}}}{\left(1+e^{\lambda\left(x+\theta+(t+1 / \Gamma(\vartheta))^{9}\left(-4 h+\left(6 h^{2}+\lambda^{2}\right) \gamma_{1}\right) / 29\right)} \mu\right) \sqrt{-\gamma_{2}-2 \gamma_{3}}} .
$$

When $4 \alpha \mu>\lambda^{2}$

$$
\begin{aligned}
& \mathscr{F}_{7}(x, t)=\frac{e^{i h x-i t \Omega} \sqrt{-3 \lambda^{2} / 2+6 \alpha \mu} \sqrt{\gamma_{1}} \operatorname{Tan}\left[1 / 2 \sqrt{-\lambda^{2}+4 \alpha \mu}\left(x+\theta+(t+1 / \Gamma(\vartheta))^{9}\left(-4 h+\left(6 h^{2}+\lambda^{2}-4 \alpha \mu\right) \gamma_{1}\right) / 2 \vartheta\right)\right]}{\sqrt{-\gamma_{2}-2 \gamma_{3}}}, \\
& \mathscr{F}_{8}(x, t)=\frac{e^{i h x-i t \Omega} \sqrt{-3 \lambda^{2} / 2+6 \alpha \mu} \operatorname{Cot}\left[1 / 2 \sqrt{-\lambda^{2}+4 \alpha \mu}\left(x+\theta+(t+1 / \Gamma(\vartheta))^{9}\left(-4 h+\left(6 h^{2}+\lambda^{2}-4 \alpha \mu\right) \gamma_{1}\right) / 2 \vartheta\right)\right] \sqrt{\gamma_{1}}}{\sqrt{-\gamma_{2}-2 \gamma_{3}}},
\end{aligned}
$$

Additionally, the optical solitary wave solutions of the Atangana conformable fractional nonlinear Schrödinger equation are given based on family II by
When $\lambda=0$, for $\alpha \mu>0$,

$$
\begin{gathered}
\mathscr{F}_{9}(x, t)=\frac{\sqrt{6} \mathrm{e}^{\mathrm{i} h x-\mathrm{i} t \Omega} \sqrt{\alpha \mu} \operatorname{Cot}\left[\sqrt{\alpha \mu}\left(x+\theta+(t+1 / \Gamma(\vartheta))^{\vartheta}\left(-4 h+\left(6 h^{2}-4 \alpha \mu\right) \gamma_{1}\right) / 2 \vartheta\right)\right] \sqrt{\gamma_{1}}}{\sqrt{-\gamma_{2}-2 \gamma_{3}}}, \\
\mathscr{F}_{10}(x, t)=\frac{\sqrt{6} \mathrm{e}^{\mathrm{i} h x-\mathrm{i} t \Omega} \sqrt{\alpha \mu} \sqrt{\gamma_{1}} \operatorname{Tan}\left[\sqrt{\alpha \mu}\left(x+\theta+(t+1 / \Gamma(\vartheta))^{\vartheta}\left(-4 h+\left(6 h^{2}-4 \alpha \mu\right) \gamma_{1}\right) / 2 \vartheta\right)\right]}{\sqrt{-\gamma_{2}-2 \gamma_{3}}} .
\end{gathered}
$$


For $\alpha \mu<0$,

$$
\begin{aligned}
& \mathscr{F}_{11}(x, t)=-\frac{\sqrt{6} \mathrm{e}^{\mathrm{i} h x-\mathrm{i} t \Omega} \sqrt{-\alpha \mu} \operatorname{Coth}\left[\sqrt{-\alpha \mu}\left(x+(t+1 / \Gamma(\vartheta))^{9}\left(-4 h+\left(6 h^{2}-4 \alpha \mu\right) \gamma_{1}\right) / 2 \vartheta\right) \mp \log [\theta] / 2\right] \sqrt{\gamma_{1}}}{\sqrt{-\gamma_{2}-2 \gamma_{3}}}, \\
& \mathscr{F}_{12}(x, t)=-\frac{\sqrt{6} e^{i h x-i t \Omega} \sqrt{-\alpha \mu} \sqrt{\gamma_{1}} \operatorname{Tan} h\left[\sqrt{-\alpha \mu}\left(x+(t+1 / \Gamma(\vartheta))^{\vartheta}\left(-4 h+\left(6 h^{2}-4 \alpha \mu\right) \gamma_{1}\right) / 2 \vartheta\right) \mp \log [\theta] / 2\right]}{\sqrt{-\gamma_{2}-2 \gamma_{3}}} .
\end{aligned}
$$

When $4 \alpha \mu>\lambda^{2}$,

$$
\begin{aligned}
& \mathscr{F}_{13}(x, t)=\frac{\sqrt{3 / 2} \mathrm{e}^{\mathrm{i} h x-\mathrm{i} t \Omega} \lambda \sqrt{\gamma_{1}}}{\sqrt{-\gamma_{2}-2 \gamma_{3}}}-\frac{2 \sqrt{6} \mathrm{e}^{\mathrm{i} h x-\mathrm{i} t \Omega} \alpha \mu \sqrt{\gamma_{1}}}{\sqrt{-\gamma_{2}-2 \gamma_{3}}\left(\lambda-\sqrt{-\lambda^{2}+4 \alpha \mu} \operatorname{Tan}\left[1 / 2 \sqrt{-\lambda^{2}+4 \alpha \mu}\left(x+\theta+(t+1 / \Gamma(\vartheta))^{9}\left(-4 h+\left(6 h^{2}+\lambda^{2}-4 \alpha \mu\right) \gamma_{1}\right) / 2 \vartheta\right)\right]\right)}, \\
& \mathscr{F}_{14}(x, t)=\frac{\sqrt{3 / 2} \mathrm{e}^{\mathrm{i} h x-\mathrm{i} t \Omega} \lambda \sqrt{\gamma_{1}}}{\sqrt{-\gamma_{2}-2 \gamma_{3}}}-\frac{2 \sqrt{6} \mathrm{e}^{\mathrm{i} h x-\mathrm{i} t \Omega} \alpha \mu \sqrt{\gamma_{1}}}{\left(\lambda-\sqrt{-\lambda^{2}+4 \alpha \mu} \operatorname{Cot}\left[1 / 2 \sqrt{-\lambda^{2}+4 \alpha \mu}\left(x+\theta+(t+1 / \Gamma(\vartheta))^{9}\left(-4 h+\left(6 h^{2}+\lambda^{2}-4 \alpha \mu\right) \gamma_{1}\right) / 29\right)\right]\right) \sqrt{-\gamma_{2}-2 \gamma_{3}}} .
\end{aligned}
$$

2.2. Generalized Kudryashov Method. Applying this scheme to equation (4) leads to the following general solution

$$
\mathscr{G}(\boldsymbol{Z})=\frac{\sum_{i=0}^{m} a_{i} Q(Z)^{i}}{\sum_{j=0}^{n} b_{j} Q(Z)^{j}}=\frac{a_{0}+Q(Z) a_{1}+Q(Z)^{2} a_{2}}{b_{0}+Q(Z) b_{1}},
$$

where $a_{i} b_{j},(i=0,1,2, j=0,1)$ are arbitrary constants. Also, $Q(3)$ satisfies the next auxiliary equation $[26,27]$ :

$$
Q^{\prime}(3)=Q(3)^{2}-Q(3)
$$

Substituting equation (21) along (22) into equation (4) and collecting all terms with the same power of $Q(3)^{j},(j=$ $0,1, \ldots, 5,6)$ lead to a system of algebraic equations. Solving this system yields the following families.

\subsubsection{Family I}

$$
\begin{aligned}
a_{0} & =\frac{a_{1} b_{0}}{-2 b_{0}+b_{1}}, a_{2}=-\frac{2 a_{1} b_{1}}{-2 b_{0}+b_{1}}, c \\
& =\frac{1}{2}\left(4 h-\gamma_{1}-6 h^{2} \gamma_{1}\right), \gamma_{3} \\
& =\frac{-12 b_{0}^{2} \gamma_{1}+12 b_{0} b_{1} \gamma_{1}-3 b_{1}^{2} \gamma_{1}-2 a_{1}^{2} \gamma_{2}}{4 a_{1}^{2}} .
\end{aligned}
$$

\subsubsection{Family II}

$$
\begin{aligned}
a_{0} & =-\frac{a_{1}}{2}, a_{2}=-a_{1}, b_{1}=-2 b_{0}, c=2 h-2 \gamma_{1}-3 h^{2} \gamma_{1}, \gamma_{3} \\
& =\frac{-24 b_{0}^{2} \gamma_{1}-a_{1}^{2} \gamma_{2}}{2 a_{1}^{2}} .
\end{aligned}
$$

\subsubsection{Family III}

$$
\begin{aligned}
a_{0} & =-\frac{a_{1}}{4}, a_{2}=-a_{1}, b_{1}=-2 b_{0}, c=\frac{1}{2}\left(4 h-\gamma_{1}-6 h^{2} \gamma_{1}\right), \gamma_{3} \\
& =\frac{-24 b_{0}^{2} \gamma_{1}-a_{1}^{2} \gamma_{2}}{2 a_{1}^{2}} .
\end{aligned}
$$

\subsubsection{Family IV}

$$
\begin{aligned}
a_{1} & =0, a_{2}=-4 a_{0}, b_{1}=2 b_{0}, c=\frac{1}{2}\left(4 h-\gamma_{1}-6 h^{2} \gamma_{1}\right), \gamma_{3} \\
& =\frac{-3 b_{0}^{2} \gamma_{1}-2 a_{0}^{2} \gamma_{2}}{4 a_{0}^{2}} .
\end{aligned}
$$

Consequently, the optical solitary wave solutions of the Atangana conformable fractional nonlinear Schrödinger equation are given based on the above families respectively by

$$
\begin{gathered}
\mathscr{F}_{15}(x, t)=\frac{e^{i(h x-t \Omega)}\left(-A e^{x}+e^{(t+1 / \Gamma(\vartheta))^{9}\left(4 h-\gamma_{1}-6 h^{2} \gamma_{1}\right) / 2 \vartheta}\right) a_{1}}{\left(A e^{x}+e^{(t+1 / \Gamma(\vartheta))^{9}\left(4 h-\gamma_{1}-6 h^{2} \gamma_{1}\right) / 2 \vartheta}\right)\left(2 b_{0}-b_{1}\right)}, \\
\mathscr{F}_{16}(x, t)=\frac{e^{i(h x-t \Omega)}\left(A^{2} e^{2 x}+e^{2(t+1 / \Gamma(\vartheta))^{9}\left(2 h-2 \gamma_{1}-3 h^{2} \gamma_{1}\right) / \vartheta}\right) a_{1}}{2\left(-A^{2} e^{2 x}+e^{2(t+1 / \Gamma(\vartheta))^{9}\left(2 h-2 \gamma_{1}-3 h^{2} \gamma_{1}\right) / \vartheta}\right) b_{0}},
\end{gathered}
$$




$$
\mathscr{F}_{17}(x, t)=\frac{e^{i(h x-t \Omega)}\left(-A e^{x}+e^{(t+1 / \Gamma(9))^{9}\left(4 h-\gamma_{1}-6 h^{2} \gamma_{1}\right) / 29}\right) a_{1}}{4\left(A e^{x}+e^{(t+1 / \Gamma(\vartheta))^{9}\left(4 h-\gamma_{1}-6 h^{2} \gamma_{1}\right) / 29}\right) b_{0}},
$$

$\mathscr{F}_{18}(x, t)=\frac{e^{i(h x-t \Omega)}\left(-A e^{x}+e^{(t+1 / \Gamma(9))^{9}\left(4 h-\gamma_{1}-6 h^{2} \gamma_{1}\right) / 29}\right) a_{0}}{\left(A e^{x}+e^{(t+1 / \Gamma(9))^{9}\left(4 h-\gamma_{1}-6 h^{2} \gamma_{1}\right) / 29}\right) b_{0}}$.

2.3. Sech-Tanh Expansion Method. Applying this scheme to equation (4) leads to the following general solution:

$$
\begin{aligned}
\mathscr{G}(\boldsymbol{Z}) & =a_{0}+\sum_{i=1}^{n} \operatorname{Sech}(3)^{i-1}\left(a_{i} \operatorname{Sech}(3)+b_{i} \operatorname{Tanh}(3)\right) \\
& =a_{0}+\operatorname{Sech}(3) a_{1}+b_{1} \operatorname{Tanh}(3),
\end{aligned}
$$

where $a_{i} b_{j},(i=0,1, j=1)$ are arbitrary constants. Substituting equation (30) into equation (4) and collecting all terms of $\operatorname{Sech}(3), \operatorname{Sech}(3)^{2}, \operatorname{Sech}(3)^{3}, \operatorname{Tanh}(3)$, $\operatorname{Sech}(3)^{2} \operatorname{Tanh}(3)$, Sech (3)Tanh (3) lead to a system of algebraic equations. Solving this system yields the following families.

\subsubsection{Family I}

$$
\left(a_{0}=0, a_{1}=\frac{\sqrt{6} \sqrt{\gamma_{1}}}{\sqrt{\gamma_{2}+2 \gamma_{3}}}, b_{1}=0, c=2 h+\gamma_{1}-3 h^{2} \gamma_{1}\right),
$$

where $\left(\gamma_{2}+2 \gamma_{3}>0, \gamma_{1}>0\right)$.

\subsubsection{Family II}

$\left(a_{0}=0, a_{1}=0, b_{1}=\frac{\sqrt{6} \sqrt{\gamma_{1}}}{\sqrt{-\gamma_{2}-2 \gamma_{3}}}, c=2 h-2 \gamma_{1}-3 h^{2} \gamma_{1}\right)$,

where $\left(\gamma_{2}+2 \gamma_{3}<0, \gamma_{1}>0\right)$.

Consequently, the optical solitary wave solutions of the Atangana conformable fractional nonlinear Schrödinger equation are given based on the above families respectively by

$$
\begin{aligned}
& \mathscr{F}_{19}(x, t)=\frac{\sqrt{6} \mathrm{e}^{\mathrm{i}(h x-t \Omega)} \operatorname{Sech}\left[x-(t+1 / \Gamma(\vartheta))^{9}\left(2 h+\gamma_{1}-3 h^{2} \gamma_{1}\right) / \vartheta\right] \sqrt{\gamma_{1}}}{\sqrt{\gamma_{2}+2 \gamma_{3}}}, \\
& \mathscr{F}_{20}(x, t)=\frac{\sqrt{6} \mathrm{e}^{\mathrm{i}(h x-t \Omega)} \sqrt{\gamma_{1}} \operatorname{Tanh}\left[x-(t+1 / \Gamma(\vartheta))^{9}\left(2 h-2 \gamma_{1}-3 h^{2} \gamma_{1}\right) / \vartheta\right]}{\sqrt{-\gamma_{2}-2 \gamma_{3}}} .
\end{aligned}
$$

\section{Stability Property of Solutions}

This part studies the stability property of each one of the obtained solutions by using the Hamiltonian system property since the momentum $M$ in this system is given by $[36,37]$

$$
\left.M=\frac{1}{2} \int_{-l}^{l} \mathscr{G}^{2}(3) d\right\}
$$

where $\mathscr{G}(3)$ is the solution of the model. Consequently, the condition for stability of the solutions can be formulated as

$$
\frac{\partial M}{\partial c}>0
$$

where $c$ is the wave velocity. The momentum in the Hamiltonian system for equation (11) is given by

$$
\begin{aligned}
M= & \frac{1}{c^{2}}\left(c\left((-1095.1320422182555+1337.3986588468201 i)+\left(10799.999990891356+6.613092659621341 \times 10^{-14} i\right) c\right)\right. \\
& +\left((-67.49999939305316+10.602875127368723 i)-\left(63.68904259247388-1.479114197289397 \times 10^{-31} i\right) c\right) \\
& \cdot \log \left[1 .+\left(2.061153687919397 \times 10^{-9}-2.524185266461253 \times 10^{-25} i\right) e^{-18.87082757451449 c}\right] \\
& +\left((-67.49999939305316+10.602875127368723 i)+\left(63.68904259247388-1.479114197289397 \times 10^{-31} i\right) c\right)
\end{aligned}
$$




$$
\begin{aligned}
& \cdot \log \left[1 .+\left(4.851651799965657 \times 10^{8}+5.941559847405437 \times 10^{-8} i\right) e^{-18.87082757451449 c}\right] \\
& +\left((67.49999939305316-10.602875127368721 i)+\left(3.482018748651115 \times 10^{-15}\right.\right. \\
& +\left((67.49999939305316-10.602875127368721 i)+\left(3.482018748651115 \times 10^{-15}-2.524185266461253 \times 10^{-25} i\right)\right. \\
& \left.\cdot e^{\left(-1.031709266497698 \times 10^{-15}-16.849090974083545 i\right) c}\right]+(67.49999939305316-10.602875127368723 i) \\
& \cdot \log \left[1 .+\left(4.851651799965657 \times 10^{8}+5.941559847405437 \times 10^{-8} i\right) e^{\left(-1.031709266497698 \times 10^{-15}-16.849090974083545 i\right) c}\right] \\
& -\left(3.482018748651115 \times 10^{-15}+56.8656816165352 i\right) c \\
& \cdot \log \left[1 .+\left(4.851651799965657 \times 10^{8}+5.941559847405437 \times 10^{-8} i\right) e^{\left(-1.031709266497698 \times 10^{-15}-16.849090974083545 i\right) c}\right] \\
& -(67.49999939305316-10.602875127368723 i) \\
& \cdot \log \left[\operatorname{Sin}\left[(1.5707963267948966+9.999999984115489 i)-\left(8.424545487041772-5.158546332488493 \times 10^{-16} i\right) c\right]\right] \\
& +(67.49999939305316-10.602875127368723 i) \\
& \cdot \log [-1 . \operatorname{Sin}[(1.5707963267948966+9.999999984115489 i)-(0 .+9.435413787257245 i) c]] \\
& +(67.49999939305316-10.602875127368723 i) \\
& \cdot \log [\operatorname{Sin}[(1.5707963267948966+9.999999984115489 i)+(0 .+9.435413787257245 i) c]] \\
& -(67.49999939305316-10.602875127368723 i) \\
& \cdot \log \left[-1 . \operatorname{Sin}\left[(1.5707963267948966+9.999999984115489 i)+\left(8.424545487041772-5.158546332488493 \times 10^{-16} i\right) c\right]\right] \\
& -\left(3.3749999750136808-3.081487911019577 \times 10^{-33} i\right) \\
& \text { · PolyLog[2., } \left.\left(-4.851651799965657 \times 10^{8}-5.941559847405437 \times 10^{-8} i\right) e^{-18.87082757451449 c}\right] \\
& +\left(3.3749999750136808-3.081487911019577 \times 10^{-33} i\right) \\
& \left.\cdot \text { PolyLog[2., }\left(-2.061153687919397 \times 10^{-9}+2.524185266461253 \times 10^{-25} i\right) e^{-18.87082757451449 c}\right] \\
& +\left(3.3749999750136808-3.081487911019577 \times 10^{-33} i\right) \\
& \left.\cdot \text { PolyLog[2., }\left(-4.851651799965657 \times 10^{8}-5.941559847405437 \times 10^{-8} i\right) e^{\left(-1.031709266497698 \times 10^{-15}-16.849090974083545 i\right) c}\right] \\
& -\left(3.3749999750136808-3.081487911019577 \times 10^{-33} i\right) \\
& \left.\cdot \text { PolyLog }\left[2 .,\left(-2.061153687919397 \times 10^{-9}+2.524185266461253 \times 10^{-25} i\right) e^{\left(-1.031709266497698 \times 10^{-15}-16.849090974083545 i\right) c}\right]\right)
\end{aligned}
$$




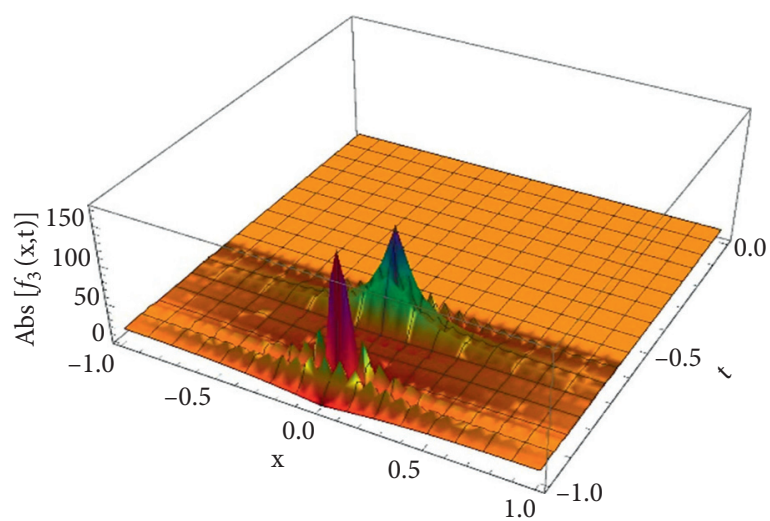

(a)

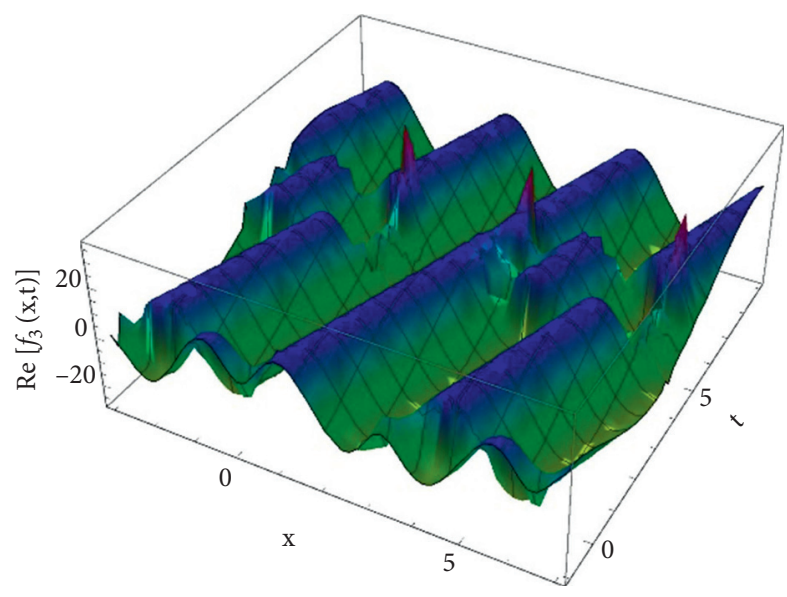

(b)

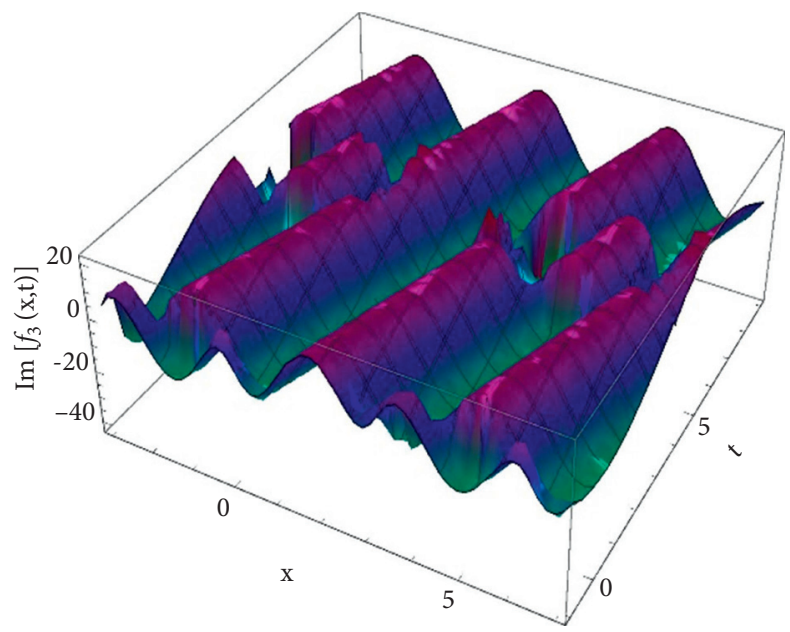

(c)

Figure 1: Optical soliton wave solutions of the absolute (a), real (b), and imaginary (c) value of equation (8) in three-dimensional view when $\left(\theta=1, \alpha=-1, \mu=4, \gamma_{1}=9, \vartheta=0.5, \gamma_{2}=-9, \gamma_{3}=4\right.$, and $\left.h=2\right)$.

and, thus

$$
\left.\frac{\partial M}{\partial c}\right|_{c=-176}>0
$$

We conclude that this solution is unstable on the interval $x \in[-5,5], t \in[-5,5] \quad$ when $\quad\left[\theta=1, \alpha=-1, \mu=4, \gamma_{1}=\right.$ $\left.9, \vartheta=0.5, \gamma_{2}=-9, \gamma_{3}=4, h=2, \lambda=0\right]$. This result shows the ability of the solutions for their application. Applying the previous steps to other obtained solutions leads to studying the stability property of all solutions. This process makes choosing suitable stable solutions to use in model applications is very easy and interesting.

\section{Representation of Obtained Solutions}

This part studies the physical interpretation of obtained solutions under a suitable choice for the values of parameters. It represents each one of the selected solutions by three- and two-dimensional plots. For each kind, we mean the shape of the solution in real and imaginary plots to show the similarities and differences between them in these cases. We have plotted each of $\mathscr{F}_{3}(x, t), \mathscr{F}_{15}(x, t)$, and $\mathscr{F}_{20}(x, t)$, Figures 1-6 under the following conditions:

Figure 1 illustrates the optical periodic solitary wave solution of $\mathscr{F}_{3}(x, t)$ in three-dimensional view when $\left[\theta=1, \alpha=-1, \mu=4, \gamma_{1}=9, \vartheta=0.5, \gamma_{2}=-9, \gamma_{3}=\right.$ $4, h=2]$ in the next interval $x \in[-5,5], t \in \in[-5,5]$ to explain the perspective view of the solution.

Figure 2 describes the optical periodic solitary wave solution of $\mathscr{F}_{3}(x, t)$ in two-dimensional view when $\left[\theta=1, \alpha=-1, \mu=4, \gamma_{1}=9, \vartheta=0.5, \gamma_{2}=-9, \gamma_{3}=\right.$ $4, h=2]$ in the next interval $x \in[-5,5], t \in \in[-5,5]$ to represent the wave propagation pattern of the wave along the $x$-axis.

Figure 3 illustrates the optical periodic solitary wave solution of $\mathscr{F}_{15}(x, t)$ in three-dimensional view when $\left[A=3, \gamma_{1}=1, A=-2, a_{1}=4, b_{0}=5, b_{1}=-1, h=2\right]$ in 

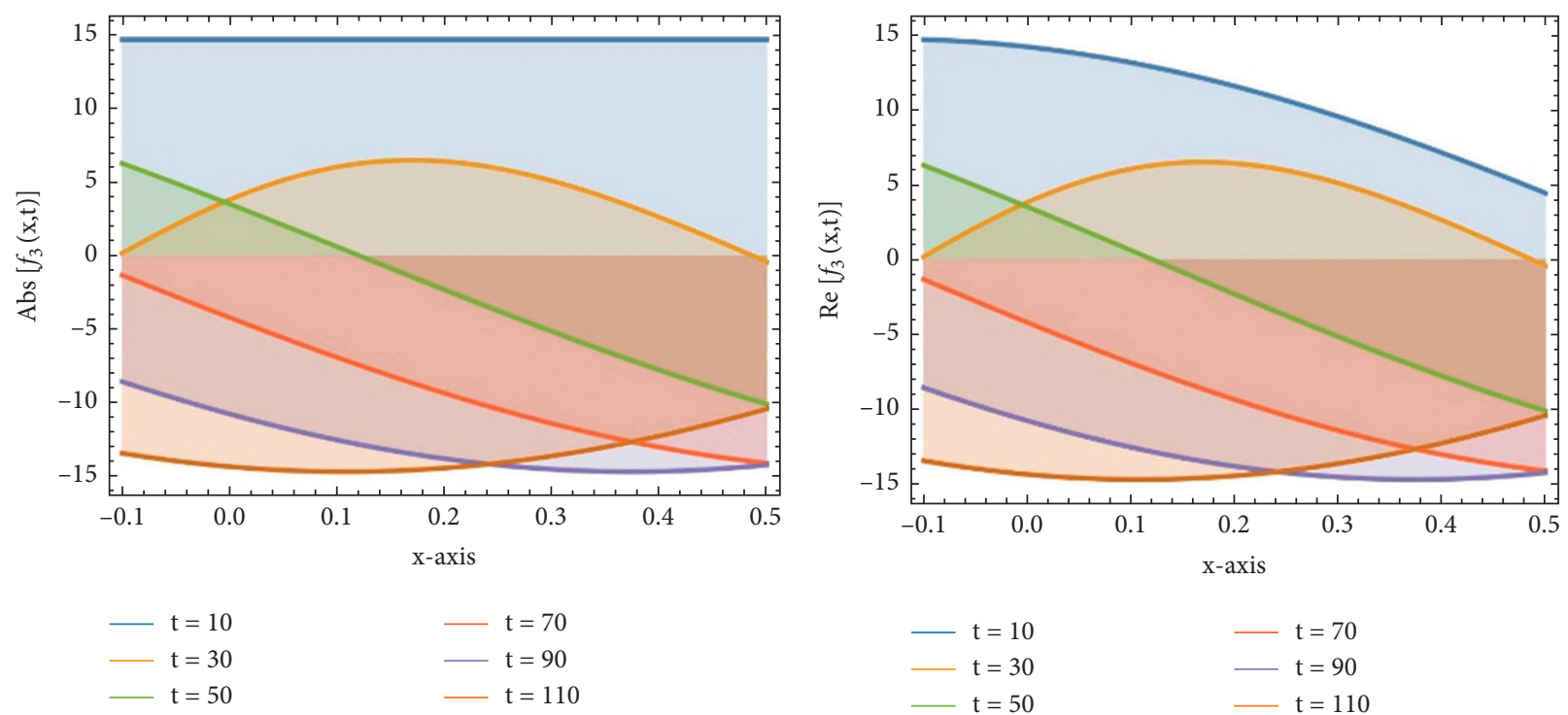

(a)

(b)

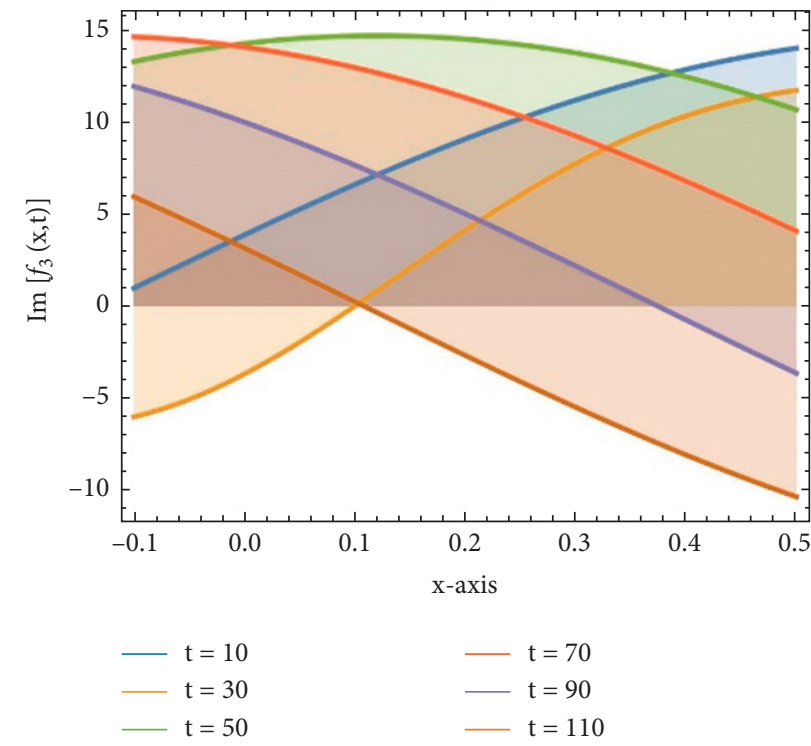

(c)

Figure 2: Optical soliton wave solutions of the absolute (a), real (b), and imaginary (c) value of equation (8) in two-dimensional view when $\left(\theta=1, \alpha=-1, \mu=4, \gamma_{1}=9, \vartheta=0.5, \gamma_{2}=-9, \gamma_{3}=4\right.$, and $\left.h=2\right)$.

the next interval $x \in[-5,5], t \in \in[-5,5]$ to explain the perspective view of the solution.

Figure 4 presents the optical periodic solitary wave solution of $\mathscr{F}_{15}(x, t)$ in two-dimensional view when $\left[A=3, \gamma_{1}=1, A=-2, a_{1}=4, b_{0}=5, b_{1}=-1, h=2\right]$ in the next interval $x \in[-5,5], t \in \in[-5,5]$ to represent the wave propagation pattern of the wave along the $x$-axis.
Figure 5 illustrates the optical periodic solitary wave solution of $\mathscr{F}_{20}(x, t)$ in three-dimensional view when $\left[\gamma_{1}=4, \vartheta=0.5, \gamma_{2}=-1, \gamma_{3}=-4, h=2\right]$ in the next interval $x \in[-5,5], t \in \in[-5,5]$ to explain the perspective view of the solution.

Figure 6 illustrates the optical periodic solitary wave solution of $\mathscr{F}_{20}(x, t)$ in two-dimensional view when 


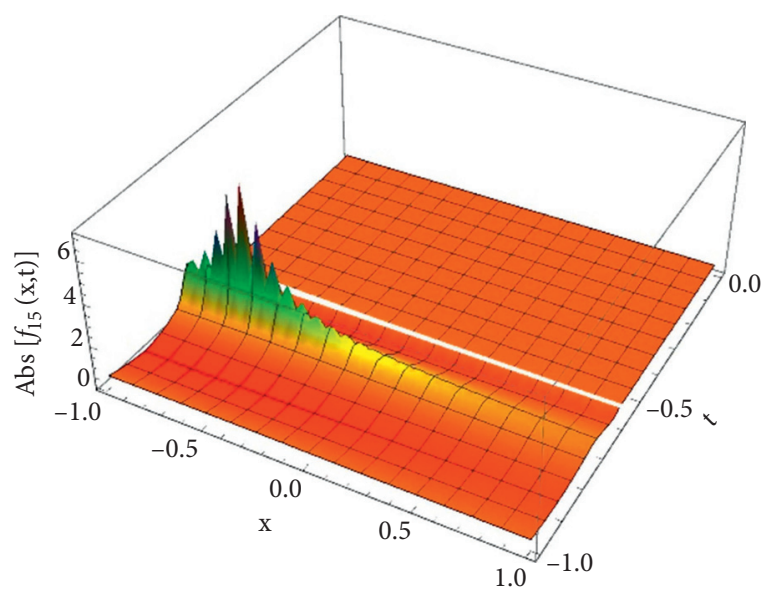

(a)

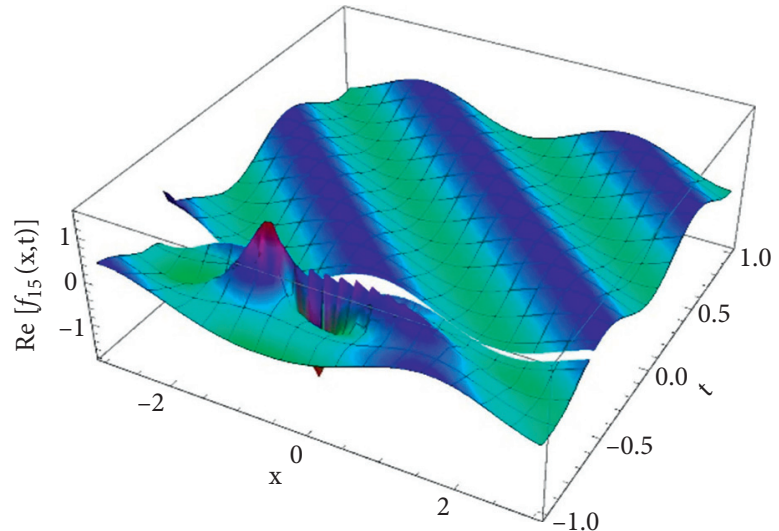

(b)

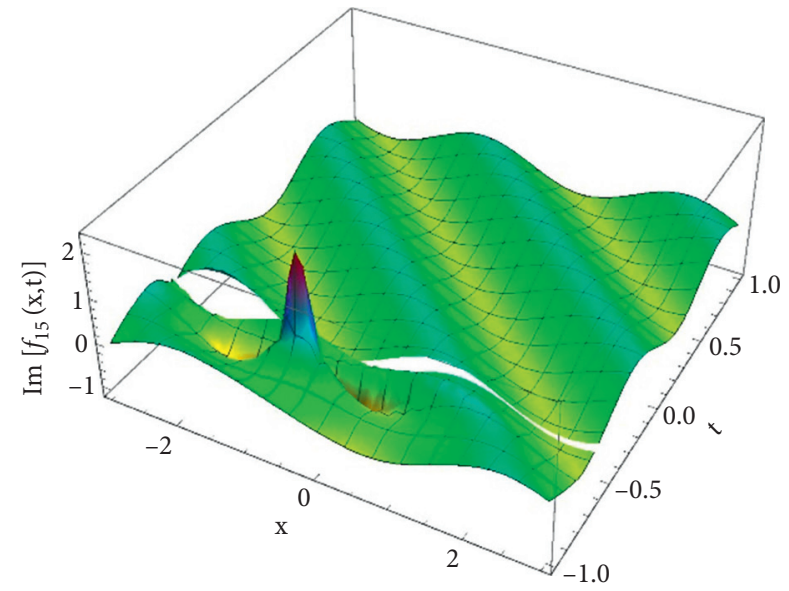

(c)

Figure 3: Optical soliton wave solutions of the absolute (a), real (b), and imaginary (c) value of equation (18) in three-dimensional view when $\left(A=3, \gamma_{1}=1, A=-2, a_{1}=4, b_{0}=5, b_{1}=-1\right.$, and $\left.h=2\right)$.

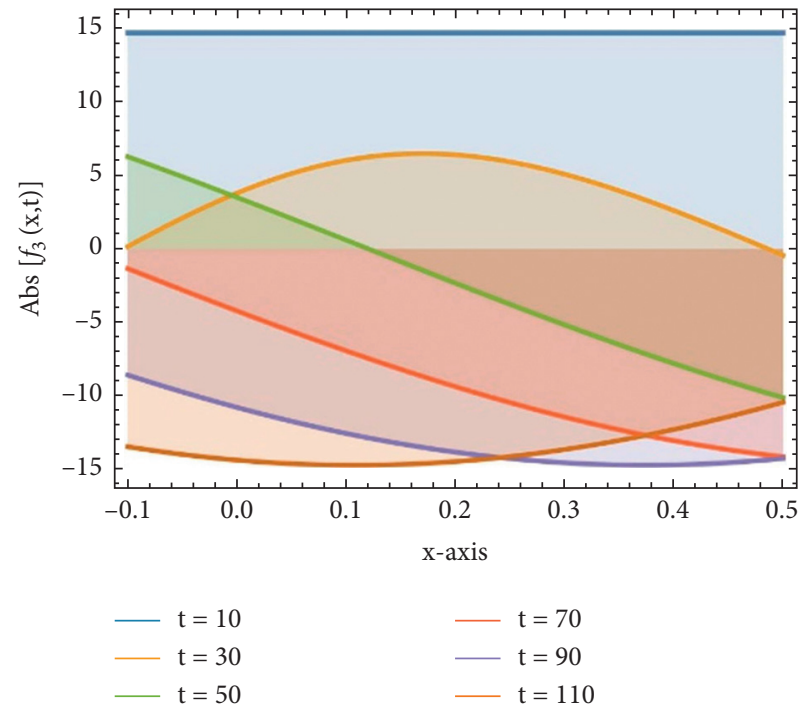

(a)

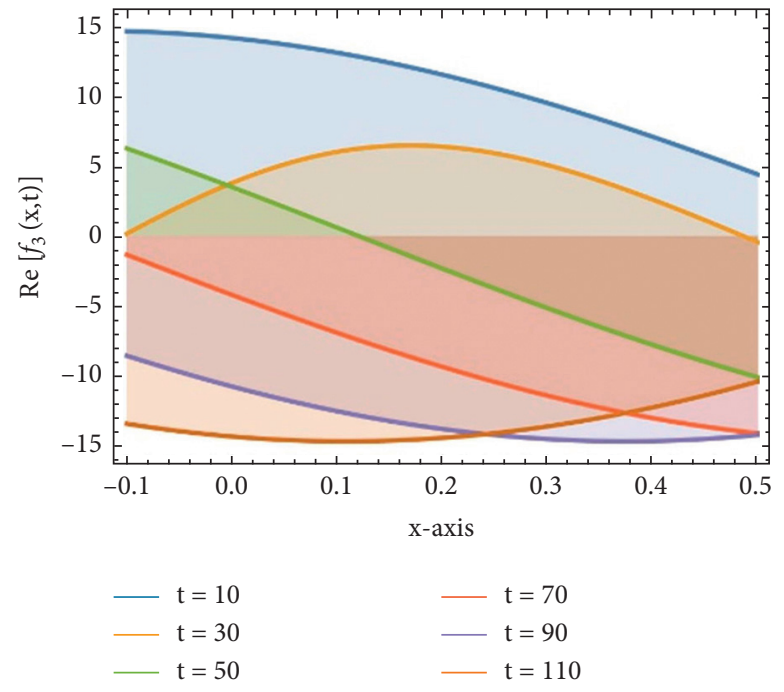

(b)

Figure 4: Continued. 


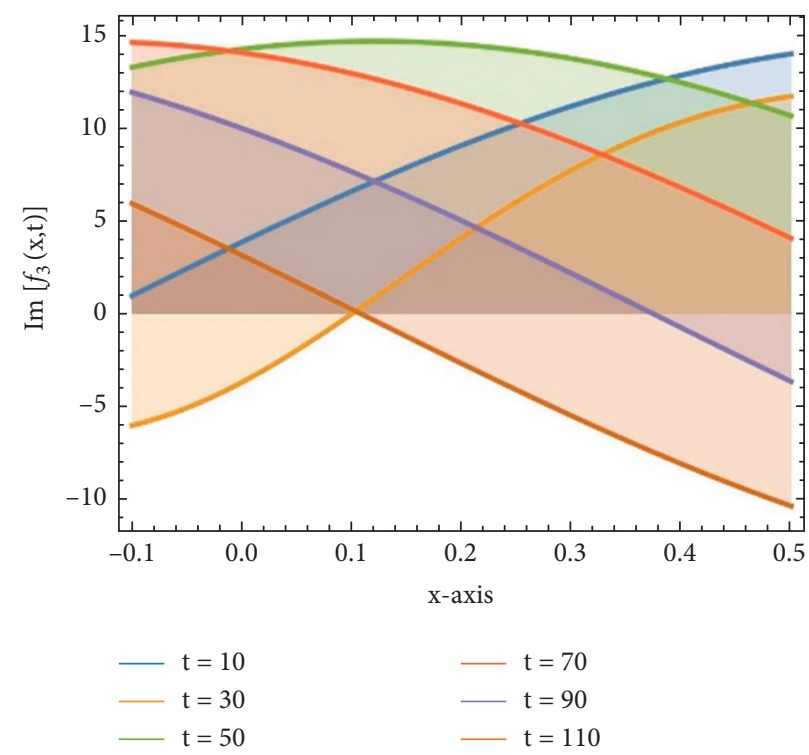

(c)

Figure 4: Optical soliton wave solutions of the absolute (a), real (b), and imaginary (c) value of equation (18) in two-dimensional view when $\left(A=3, \gamma_{1}=1, A=-2, a_{1}=4, b_{0}=5, b_{1}=-1\right.$, and $\left.h=2\right)$.

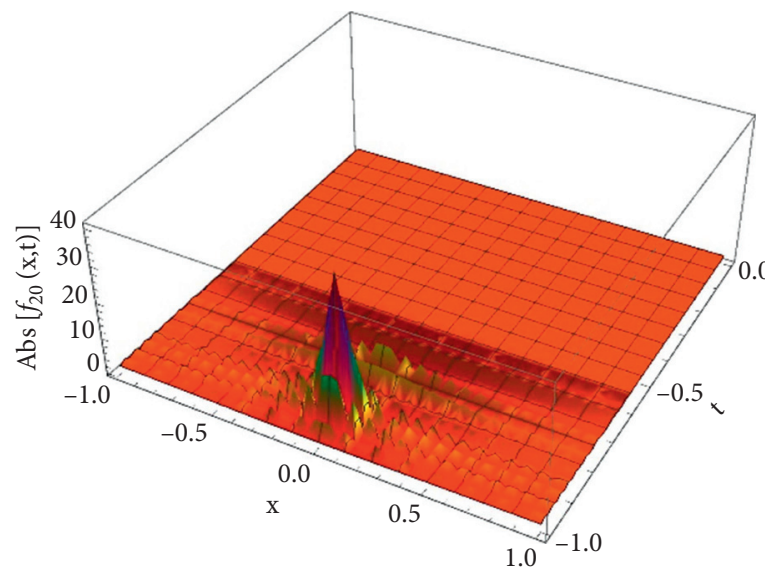

(a)

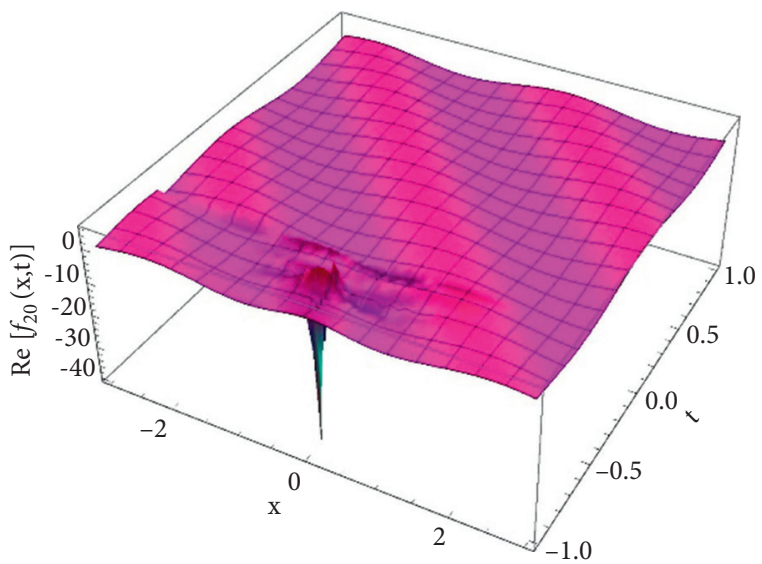

(b)

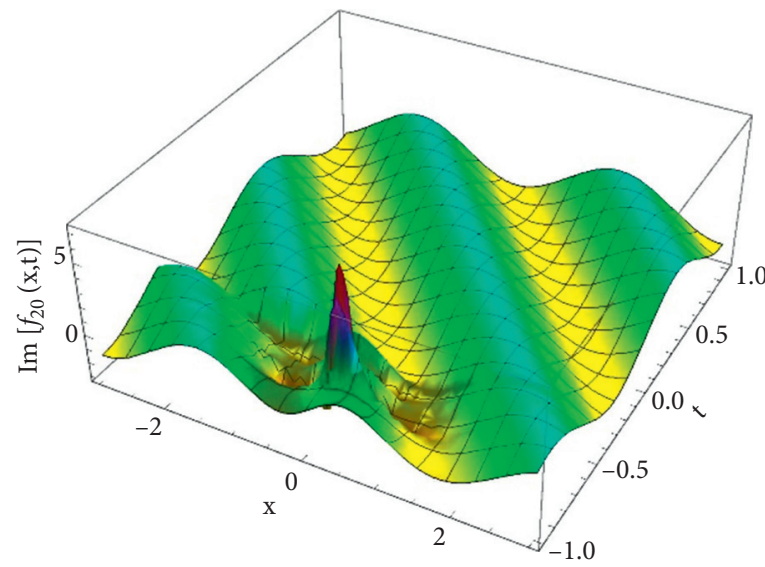

(c)

FIGURE 5: Optical soliton wave solutions of the absolute, real, and imaginary value of equation (26) in three-dimensional view $\left(\gamma_{1}=4\right.$, $\vartheta=0.5, \gamma_{2}=-1, \gamma_{3}=-4$, and $h=2$ ). 


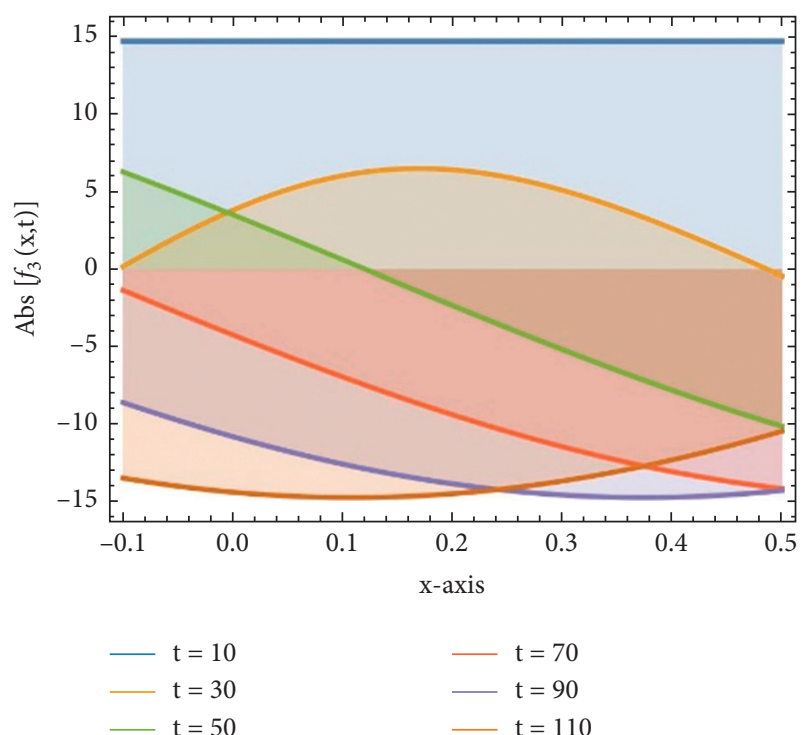

(a)

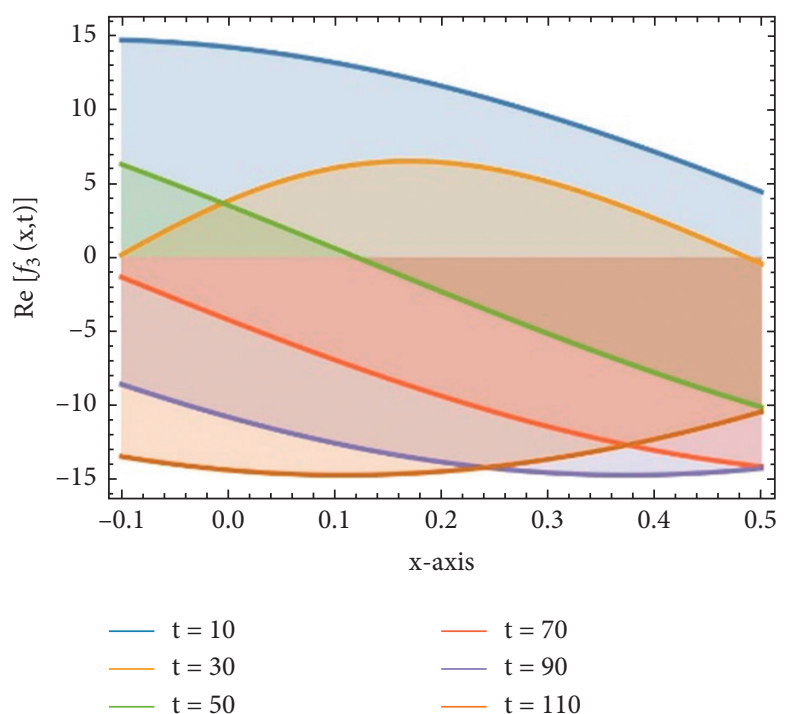

(b)

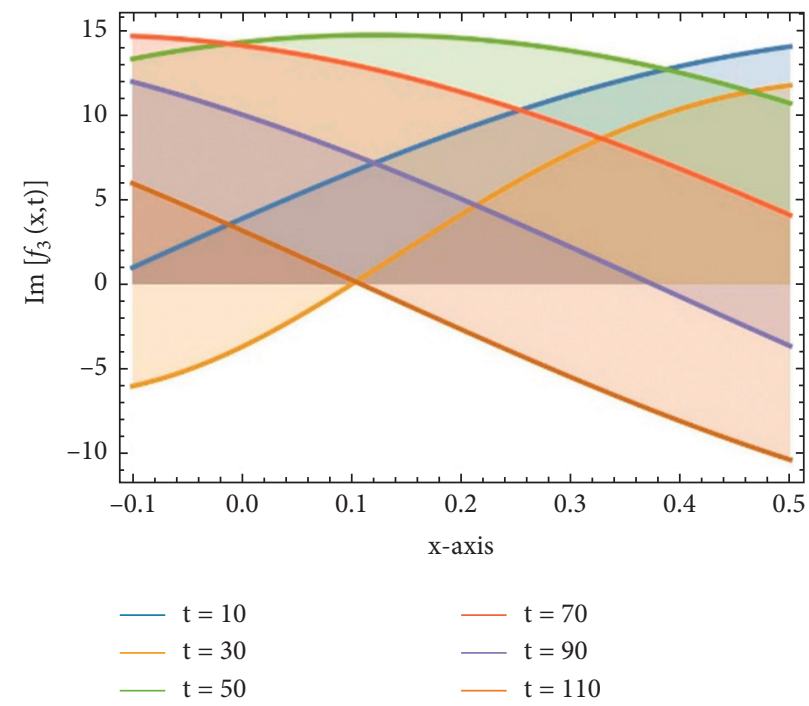

(c)

Figure 6: Optical soliton wave solutions of the absolute, real, and imaginary value of equation (26) in two-dimensional view when $\left(\gamma_{1}=4\right.$, $\vartheta=0.5, \gamma_{2}=-1, \gamma_{3}=-4$, and $h=2$ ).

$\left[\gamma_{1}=4, \vartheta=0.5, \gamma_{2}=-1, \gamma_{3}=-4, h=2\right]$ in the next interval $x \in[-5,5], t \in \in[-5,5]$ to represent the wave propagation pattern of the wave along the $x$-axis.

\section{Conclusion}

The consistent fragmental derivative concept of Atangana successfully enables us to introduce three recent computational systems for the disturbed time-fractional nonlinear Schrödinger equation. This model has been given modern wave analytical solutions. We have researched the reliability of strategies to illustrate their potential to work in the sample implementations. The robust and efficient implementation of the computing schemes has been tested, demonstrating its capacity with an integer or fractional order to handle many nonlinear partial equations.

\section{Data Availability}

No data were used to support this study.

\section{Conflicts of Interest}

The authors declare that they have no conflicts of interest.

\section{References}

[1] J. Manafian, O. A. Ilhan, O. Alp Ilhan, and S. Abid Mohammed, "Forming localized waves of the nonlinearity of the DNA dynamics arising in oscillator-chain of PeyrardBishop model," AIMS Mathematics, vol. 5, no. 3, pp. 2461-2483, 2020.

[2] O. A. Ilhan, J. Manafian, A. A. Alizadeh, and H. M. Baskonus, "New exact solutions for nematicons in liquid crystals by the 
$\tan (\phi / 2)$-expansion method arising in fluid mechanics," The European Physical Journal Plus, vol. 135, no. 3, pp. 1-19, 2020.

[3] J. Manafian, O. A. Ilhan, K. K. Ali, and S. Abid, "Cross-kink wave solutions and semi-inverse variational method for $(3+$ 1)-dimensional potential-YTSF equation," East Asian Journal on Applied Mathematics, vol. 10, no. 3, pp. 549-565, 2020.

[4] Q. Lu, O. A. Ilhan, J. Manafian, and L. Avazpour, "Multiple rogue wave solutions for a variable-coefficient Kadomtsev-Petviashvili equation," International Journal of Computer Mathematics, vol. 98, no. 7, pp. 1457-1473, 2020.

[5] M. Dehghan, J. Manafian, and A. Saadatmandi, "Analytical treatment of some partial differential equations arising in mathematical physics by using threexp-function method," International Journal of Modern Physics B, vol. 25, no. 22, pp. 2965-2981, 2011.

[6] P. Wan, J. Manafian, H. F. Ismael, and S. A. Mohammed, "Investigating one-, two-, and triple-wave solutions via multiple exp-function method arising in engineering sciences," Advances in Mathematical Physics, vol. 2020, Article ID 8018064, 18 pages, 2020.

[7] C. Yue, D. Lu, M. M. Khater, A. H. Abdel-Aty, W. Alharbi, and R. A. Attia, "On explicit wave solutions of the fractional nonlinear DSW system via the modified khater method," Fractals, vol. 28, no. 8, Article ID 2040034, 2020.

[8] T. A. Sulaiman, U. Younas, A. Yusuf, M. Younis, M. Bilal, and Shafqat-Ur-Rehman, "Extraction of new optical solitons and MI analysis to three coupled Gross-Pitaevskii system in the spinor Bose-Einstein condensate," Modern Physics Letters B, vol. 35, no. 6, Article ID 2150109, 2021.

[9] A. H. Abdel-Aty, M. M. Khater, R. A. Attia, M. Abdel-Aty, and $\mathrm{H}$. Eleuch, "On the new explicit solutions of the fractional nonlinear space-time nuclear model," Fractals, vol. 28, no. 8, Article ID 2040035, 2020.

[10] M. Alquran, F. Yousef, F. Alquran, T. A. Sulaiman, and A. Yusuf, "Dual-wave solutions for the quadratic-cubic conformable-Caputo time-fractional Klein-Fock-Gordon equation," Mathematics and Computers in Simulation, vol. 185, pp. 62-76, 2021.

[11] M. Alquran, I. Jaradat, A. Yusuf, and T. A. Sulaiman, "Heartcusp and bell-shaped-cusp optical solitons for an extended two-mode version of the complex Hirota model: application in optics," Optical and Quantum Electronics, vol. 53, no. 1, pp. 1-13, 2021.

[12] J. Dan, S. Sain, A. Ghose-Choudhury, and S. Garai, "Solitary wave solutions of nonlinear PDEs using Kudryashov's R function method," Journal of Modern Optics, vol. 67, no. 19, pp. 1499-1507, 2021.

[13] J. Li, R. A. M. Attia, M. M. A. Khater, and D. Lu, "The new structure of analytical and semi-analytical solutions of the longitudinal plasma wave equation in a magneto-electroelastic circular rod," Modern Physics Letters B, vol. 34, no. 12, Article ID 2050123, 2020.

[14] H. Ahmad, T. A. Khan, P. S. Stanimirovic, and I. Ahmad, "Modified variational iteration technique for the numerical solution of fifth order KdV type equations," Journal of Applied and Computational Mechanics, vol. 6, pp. 1220-1227, 2020.

[15] K. S. Al-Ghafri and E. V. Krishnan, "Comment on:"Exact analytic solitary wave solutions for the RKL model"[math. Comput. Simulation 80 (4)(2009) 849-854]," Mathematics and Computers in Simulation, vol. 181, pp. 113-116, 2021.

[16] M. H. Srivastava, H. Ahmad, I. Ahmad, P. Thounthong, and N. M. Khan, "Numerical simulation of three-dimensional fractional-order convection-diffusion PDEs by a local meshless method," Thermal Science, vol. 25, pp. 347-358, 2020.

[17] J.-H. He, N. Qie, and C.-H. He, "Solitary waves travelling along an unsmooth boundary," Results in Physics, vol. 24, Article ID 104104, 2021.

[18] R. M. Jena and S. Chakraverty, "Residual power series method for solving time-fractional model of vibration equation of large membranes," Journal of Applied and Computational Mechanics, vol. 5, no. 4, pp. 603-615, 2019.

[19] C. Yue, M. M. A. Khater, R. A. M. Attia, and D. Lu, "Computational simulations of the couple Boiti-Leon-Pempinelli (BLP) system and the (3+1)-dimensional KadomtsevPetviashvili (KP) equation," AIP Advances, vol. 10, no. 4, Article ID 045216, 2020.

[20] V. S. Guliyev, A. Ali, S. . Çelik, and M. N. Omarova, "Higher Order Riesz Transforms Related to Schrodinger Type Operator on Local Generalized Morrey Spaces," Journal of Pure and Applied Mathematics, vol. 10, no. 1, pp. 58-75, 2019.

[21] D. Baleanu and J. Hassan Kamil, "A novel approach for korteweg-de vries equation of fractional order," Journal of Applied and Computational Mechanics, vol. 5, no. 2, pp. 192-198, 2019.

[22] M. M. A. Khater, R. A. M. Attia, and D. Lu, "Computational and numerical simulations for the nonlinear fractional Kolmogorov-Petrovskii-Piskunov (FKPP) equation," Physica Scripta, vol. 95, no. 5, Article ID 055213, 2020.

[23] C. Liu, H. Wang, and Z. Feng, "Global solution for a sixthorder nonlinear Schrödinger equation," Journal of Mathematical Analysis and Applications, vol. 490, no. 2, Article ID 124327, 2020.

[24] G. la Forgia, T. Tokyay, C. Adduce, and G. Constantinescu, "Bed shear stress and sediment entrainment potential for breaking of internal solitary waves," Advances in Water Resources, vol. 135, Article ID 103475, 2020.

[25] N. Faraz, M. Sadaf, G. Akram, I. Zainab, and Y. Khan, "Effects of fractional order time derivative on the solitary wave dynamics of the generalized ZK-Burgers equation," Results in Physics, vol. 25, Article ID 104217, 2021.

[26] S. El-Ganaini and E. M. E. Zayed, "Short comment on "the extended simplest equation method and the (G'/G-1/G)expansion method"," Optik, vol. 206, Article ID 164258, 2020.

[27] O. A. Arqub and M. Al-Smadi, "Fuzzy conformable fractional differential equations: novel extended approach and new numerical solutions," Soft Computing, vol. 24, no. 16, pp. 12501-12522, 2020.

[28] A.-A. Hyder and M. A. Barakat, "General improved Kudryashov method for exact solutions of nonlinear evolution equations in mathematical physics," Physica Scripta, vol. 95, no. 4, Article ID 045212, 2020.

[29] S. F. Tian, X. F. Wang, T. T. Zhang, and W. H. Qiu, "Stability analysis, solitary wave and explicit power series solutions of a $(2+1)$-dimensional nonlinear Schrödinger equation in a multicomponent plasma," International Journal of Numerical Methods for Heat \& Fluid Flow, vol. 31, no. 5, pp. 1732-1748, 2021.

[30] D. D. Ganji and M. Abdollahzadeh, "Exact travelling solutions for the Lax's seventh-order KdV equation by sech method and rational exp-function method," Applied Mathematics and Computation, vol. 206, no. 1, pp. 438-444, 2008.

[31] M. Guo, H. Dong, J. Liu, and H. Yang, “The time-fractional mZK equation for gravity solitary waves and solutions using sech-tanh and radial basic function method," Nonlinear Analysis: Modelling and Control, vol. 24, no. 1, pp. 1-19, 2019. 
[32] A. Yildirim, "An algorithm for solving the fractional nonlinear schrödinger equation by means of the homotopy perturbation method," International Journal of Nonlinear Sciences and Numerical Stimulation, vol. 10, no. 4, pp. 445450, 2009.

[33] M. Ilie, J. Biazar, and Z. Ayati, "Resonant solitons to the nonlinear Schrödinger equation with different forms of nonlinearities," Optik, vol. 164, pp. 201-209, 2018.

[34] M. Younis, H. ur Rehman, S. T. R. Rizvi, and S. A. Mahmood, "Dark and singular optical solitons perturbation with fractional temporal evolution," Superlattices and Microstructures, vol. 104, pp. 525-531, 2017.

[35] S. Kumar, A. Kumar, and D. Baleanu, "Two analytical methods for time-fractional nonlinear coupled BoussinesqBurger's equations arise in propagation of shallow water waves," Nonlinear Dynamics, vol. 85, no. 2, pp. 699-715, 2016.

[36] Y. Z. Sun, Q. J. Liu, Y. H. Song, and T. L. Shen, "Hamiltonian modelling and nonlinear disturbance attenuation control of TCSC for improving power system stability," IEE Proceedings-Control Theory and Applications, vol. 149, no. 4, pp. 278-284, 2002.

[37] M. Ramírez Barrios, J. Collado, and F. Dohnal, "Stability of coupled and damped Mathieu equations utilizing symplectic properties," in Nonlinear Dynamics of Structures, Systems and Devices, pp. 137-145, Springer, Cham, Switzerland, 2020. 